\title{
Inhibition of Tumor-Induced Angiogenesis and Matrix-Metalloproteinase Expression in Confrontation Cultures of Embryoid Bodies and Tumor Spheroids by Plant Ingredients Used in Traditional Chinese Medicine
}

\author{
Maria Wartenberg, Paula Budde, Markus de Mareés, Frank Grünheck, \\ Suk Ying Tsang, Yu Huang, Zhen-Yu Chen, Jürgen Hescheler, and Heinrich Sauer \\ Department of Neurophysiology (MW, PB, MdM, FG, JH, HS), University of Cologne, Cologne, Germany; and \\ Departments of Physiology (SYT, YH) and Biochemistry (Z-YC), Chinese University of Hong Kong, Hong Kong, \\ P.R. China
}

\begin{abstract}
SUMMARY: Tumor-induced angiogenesis is a prerequisite for excessive tumor growth. Blood vessels invade the tumor tissue after degradation of the extracellular matrix scaffold by matrix metalloproteinases (MMPs). Inhibition of MMPs has been therefore suggested to be a useful tool to abolish neoangiogenesis of solid tumors. In the present study, antioxidative plant ingredients used in traditional Chinese medicine were investigated for their capacity to down-regulate MMP expression and to inhibit angiogenesis in embryonic stem cell-derived embryoid bodies and tumor-induced angiogenesis in confrontation cultures consisting of embryoid bodies and multicellular DU-145 prostate tumor spheroids. Embryoid bodies transiently expressed MMP-1, MMP-2, and MMP-9 during the time of differentiation of capillary-like structures. In confrontation cultures, MMP expression was increased compared with control tumor spheroids and embryoid bodies cultivated separately. The increased expression of MMPs in confrontation cultures was a result of elevated levels of reactive oxygen species (ROS) upon confrontation culture and was totally abolished in the presence of the free radical scavenger vitamin E. Incubation of embryoid bodies with baicalein, epicatechin, berberine, and acteoside, which are herbal ingredients used in traditional Chinese medicine, significantly inhibited angiogenesis in embryoid bodies and decreased intracellular ROS levels. Tumor-induced angiogenesis in confrontation cultures was totally abolished in the presence of the free radical scavenger vitamin E. Because herbal ingredients down-regulated MMP expression, we conclude that ROS generated during confrontation culture induce the expression of MMPs that are necessary for endothelial cell invasion into the tumor tissue. (Lab Invest 2003, 83:87-98).
\end{abstract}

\begin{abstract}
$T \mathrm{t}$ is well known that tumors cannot grow beyond a 1 few millimeters without blood supply from the host tissue, a process called tumor-induced angiogenesis. This observation led to the development of antiangiogenic therapy by Folkman and colleagues (Folkman, 1971; Folkman et al, 1971), which is based on the assumption that inhibition of tumor-induced angiogenesis would deprive the growing tumor of nutrients and oxygen supplied by the host circulation and, consequently, would retard or even abolish tumor growth. During recent years a variety of proangiogenic and antiangiogenic agents have been found, many of them now being tested in clinical trials. However, the
\end{abstract}

DOI: 10.1097/01.LAB.0000049348.51663.2F

Received September 21, 2002.

Supported by Competence Network Stem Cell Research, Nordrhein-Westfalen.

Address reprint requests to: Dr. H. Sauer, Department of Neurophysiology, University of Cologne, Robert-Koch-Str. 39, D-50931 Cologne, Germany. E-mail:heinrich.sauer@uni-koeln.de molecular mechanisms of action of most compounds that are active in inhibiting angiogenesis are currently unknown.

There is increasing evidence for an involvement of reactive oxygen species (ROS) in the regulation of angiogenesis. Although high levels of ROS (eg, the highly reactive hydroxyl radical) have been demonstrated to exert antiangiogenic effects (Sauer et al, 2000a) and to promote arteriosclerosis and endothelial cell death (Angkeow et al, 2002; Heitzer et al, 2001), very low levels of ROS have been demonstrated to be involved in signal transduction cascades that regulate endothelial cell growth and migration (Abid et al, 2000; Van Wetering et al, 2002). This observation has led to the suggestion that ROS are mediating the angiogenic switch, thereby increasing the vascularity of tumors and inducing molecular markers of angiogenesis (Arbiser et al, 2002). Furthermore, ROS have been demonstrated to be involved in the progression of tumor-induced angiogenesis because matrix metalloproteinase (MMP) expression is regulated by the intracellular redox state (Inoue et al, 
2001; Yoon et al, 2002). MMPs are essential for endothelial cell ingression into the tumor tissue because they degrade the extracellular matrix of the tumor cells and free the way for migrating endothelial cells.

The involvement of ROS as signaling molecules that regulate essential endothelial signal transduction cascades has prompted the notion that agents that lower the intracellular redox state (eg, free radical scavengers) may be utilized as antiangiogenic agents. Several agents that are utilized in traditional Chinese herbal medicine have been shown to act as free radical scavengers (Choi et al, 2001; Harada et al, 1999; Shao et al, 2002; Terao, 1999; Wong et al, 2001a; Zhang et al, 2000) and may be useful to inhibit angiogenesis. These include baicalein, which has been purified from the dry roots of Scutellaria baicalensis Georgi (common name: Huangqin); acteoside, which has been isolated from the leaves of Eremophila alternifolia and Ligustrum purpurascens; berberine, extracted from Arcangelisia flava (L.) Merr. and Berberis crataegina DC; and epicatechin, which is a major constituent of green tea.

The present study was undertaken to evaluate the potency of the ingredients of herbs used in traditional Chinese medicine and of the free radical scavenger vitamin $E$ in angioprevention and to test whether the applied agents interfered with the expression levels of MMPs that are involved in the promotion of angiogenesis. Our study used embryoid bodies grown from pluripotent murine embryonic stem (ES) cells. This three-dimensional in vitro model differentiates functional blood vessel-like structures and has been previously demonstrated by us to be an excellent model for routine screening of antiangiogenic agents (Wartenberg et al, 1998). Furthermore, changes in the intracellular redox state and in MMP expression and angiogenesis were studied in confrontation cultures of multicellular tumor spheroids and embryoid bodies. Confrontation cultures of tumor spheroids and embryoid bodies have been recently established by us and have been successfully used to study the time course and the expression of proangiogenic molecules (eg, vascular endothelial growth factor [VEGF] during tumor-induced angiogenesis; Wartenberg et al, 2001a). Tumor-induced angiogenesis in confrontation cultures is accompanied by an elevation of intracellular ROS levels and up-regulation of MMP-1, MMP-2, and MMP-9. The herbal ingredients acteoside, baicalein, berberine, and epicatechin efficiently inhibited angiogenesis in embryoid bodies and down-regulated MMP expression. We therefore conclude that the antiangiogenic effects of these compounds are mediated through the inhibition of MMP expression.

\section{Results}

\section{Expression of MMPs During the Time Course of Vasculogenesis in ES Cell-Derived Embryoid Bodies}

It has been previously reported that vasculogenesis and angiogenesis occur in embryoid bodies grown in suspension culture from ES cells (Vittet et al, 1996; Wang et al, 1992; Wartenberg et al, 1998). However, the expression of MMPs, which are essential for the progression of blood vessel growth, has not yet been investigated. We examined the expression levels of MMP-1, MMP-2, and MMP-9 during the time course of embryoid body differentiation (ie, Day 2 through Day 10; Fig. 1). It was observed that MMP expression transiently increased during embryoid body culture, with maximum expression at Day 8 of cell culture $(660$ $\pm 140 \%, 632 \pm 116 \%$, and $262 \pm 69 \%$ for MMP-1, MMP-2, and MMP-9, respectively) (immunofluorescence at Day 2 set to $100 \%)(n=3)$. The time course of MMP expression closely coincided with the time course of vascular development in embryoid bodies, which was maximum at Day 8 of embryoid body culture (Wartenberg et al, 1998).

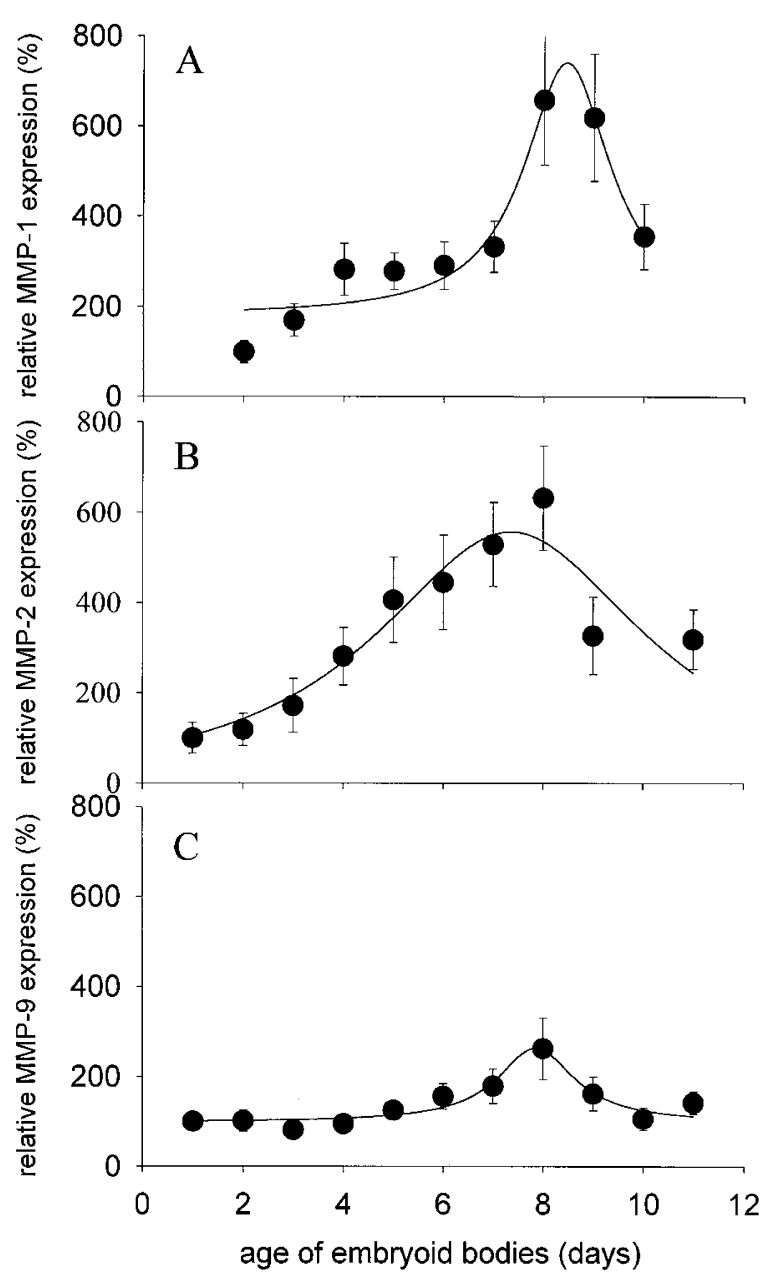

Figure 1.

Expression of matrix metalloproteinase 1 (MMP-1) (A), MMP-2 (B), and MMP-9 (C) during the time course of differentiation of embryoid bodies grown from pluripotent embryonic stem cells. MMP expression was evaluated by quantitative immunohistochemistry in whole mount embryoid bodies. Note that MMP expression transiently increased during embryoid body differentiation. The expression level of MMP-9 was significantly lower compared with MMP-1 and MMP-2. 


\section{Expression of MMPs in Confrontation Cultures of Multicellular Tumor Spheroids and Embryoid Bodies}

We have recently investigated tumor-induced angiogenesis in confrontation cultures of multicellular prostate tumor spheroids and embryoid bodies. It was observed that upon confrontation culture, capillarylike structures were invading the tumor tissue, which could be inhibited by the $\mathrm{Flk}-1$ receptor antagonist SU5614 (Wartenberg et al, 2001a). Tumor-induced angiogenesis should be paralleled by an increase in the activity and/or expression of MMPs that degrade the extracellular matrix of tumor cells, thereby allowing the ingression of the endothelial cells that form the capillary wall. Therefore MMP expression was investigated in confrontation cultures and in single tumor spheroids and embryoid bodies. It was observed that upon confrontation culture, a transient rise in the expression of MMP-1, MMP-2, and MMP-9 was evident in the tissue of multicellular tumor spheroids and in embryoid bodies (Fig. 2, A to C). Interestingly, the

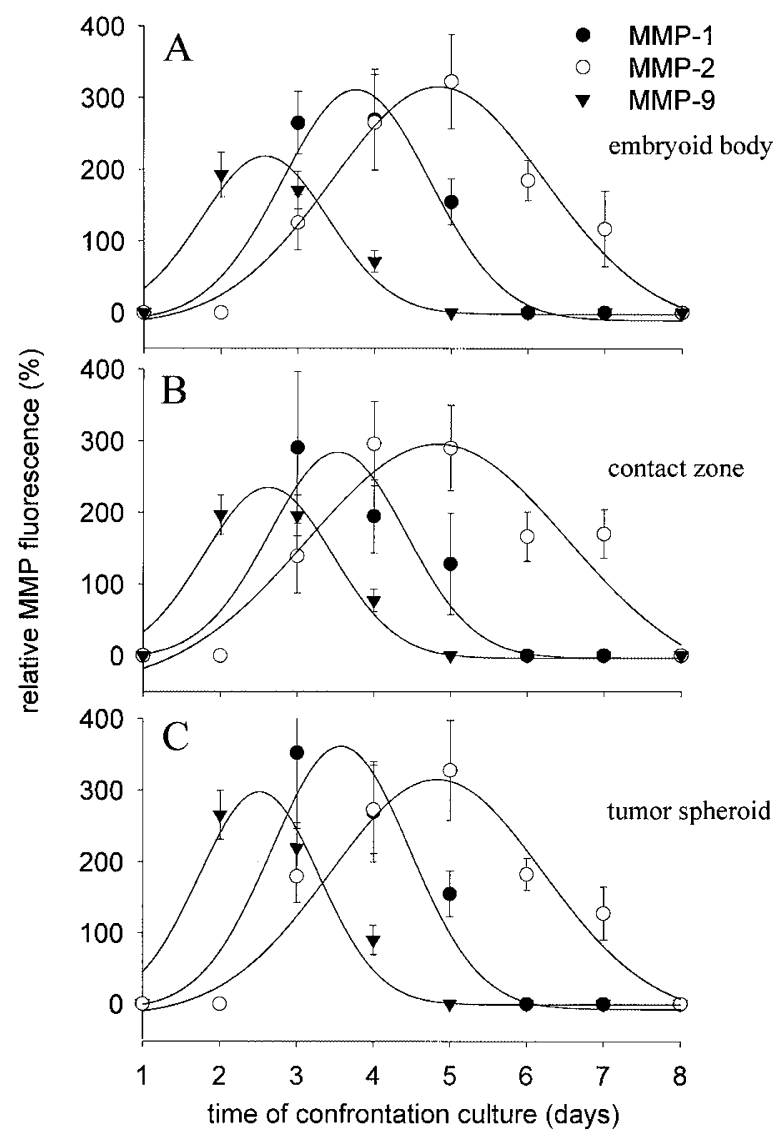

Figure 2.

Transient expression of MMP-1, MMP-2, and MMP-9 in confrontation cultures of embryoid bodies and multicellular tumor spheroids. MMP expression was investigated in different regions of the confrontation cultures, such as the embryoid body region (A), the contact zone between the embryoid body and the multicellular tumor spheroid $(B)$, and the tumor spheroid region $(C)$ of the confrontation culture. For confrontation culture, 5-day-old embryoid bodies were used. MMP fluorescence was evaluated on Day 1 (24 hours of confrontation culture was set to $0 \%$ ). Note that confrontation culture resulted in a transient increase in the expression of MMPs with a distinct time course for MMP-1, MMP-2, and MMP-9. up-regulation of MMPs during confrontation culture followed a distinct time frame. The first MMP to be up-regulated was MMP-9, which reached maximum expression at Day 2 of confrontation culture $(n=3)$. The transient up-regulation of MMP-9 was followed by MMP-1, which reached maximum expression on Days 3 and 4 of confrontation culture $(n=3)$, and MMP-2, which reached maximum expression on Day 5 of confrontation culture $(n=3)$. The increase in MMP expression in confrontation culture was totally abolished upon preincubation with the free radical scavenger vitamin $\mathrm{E}(100 \mu \mathrm{M})$ (Fig. 3, A to C) $(n=3)$, indicating that MMP expression was dependent on the intracellular redox state of the tissue.

\section{Elevation of ROS Upon Confrontation Culture of Multicellular Tumor Spheroids and Embryoid Bodies}

It has been previously reported that the expression of MMPs is regulated by the intracellular redox state. In this respect endogenous addition of ROS (eg, hydro-

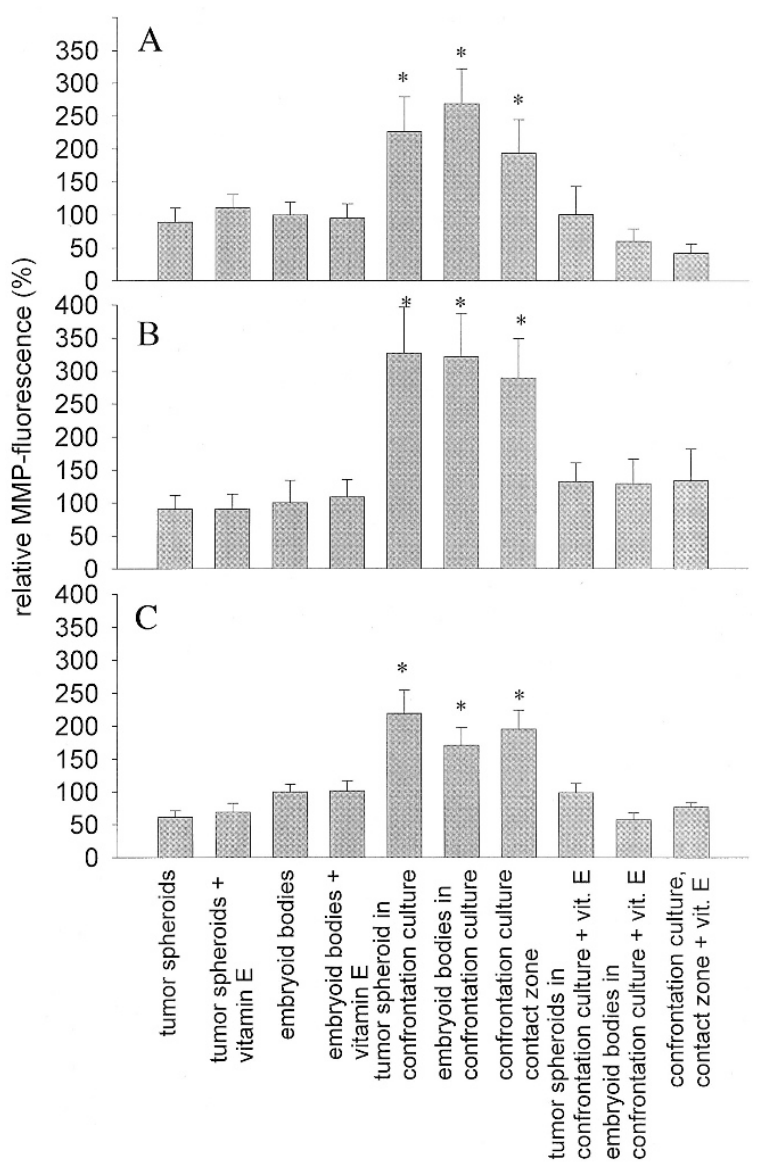

Figure 3.

Effects of the free radical scavenger vitamin $E$ on the expression of MMP-1 (A), MMP-2 (B), and MMP-9 (C) in embryoid bodies and multicellular tumor spheroids cultivated either separately or in confrontation culture. MMP immunofluorescence in embryoid bodies was set to $100 \%$. MMP-1 expression was evaluated on Day 3 of confrontation culture, MMP-2 expression was evaluated on Day 5 of confrontation culture, and MMP-9 expression was evaluated on Day 2 of confrontation culture. Note that the increase in MMP expression observed upon confrontation culture was completely abolished in the presence of the free radical scavenger vitamin $\mathrm{E}$. 
gen peroxide) resulted in up-regulation of MMP expression (Belkhiri et al, 1997; Brenneisen et al, 1997; Gurjar et al, 2001). Because confrontation culture of multicellular tumor spheroids and embryoid bodies resulted in a transient up-regulation of MMP expression in the tissue of multicellular tumor spheroids and of embryoid bodies it was hypothesized that confrontation culture may induce an elevation of the intracellular redox state. To investigate this, confrontation cultures and single multicellular tumor spheroids and embryoid bodies were labeled with the redoxsensitive fluorescence dye $\mathrm{H}_{2}$ DCF-DA $\left(\mathrm{H}_{2} 2^{\prime}, 7^{\prime}-\right.$ dichlorofluorescein diacetate), and intracellular ROS levels were evaluated by quantification of the fluorescent oxidation product DCF (Fig. 4). It was demonstrated that confrontation culture (2 days) significantly resulted in an approximately 5-fold increase in ROS in embryoid bodies compared with embryoid bodies cultivated in the absence of tumor spheroids. Likewise an elevation in intracellular ROS levels was achieved in tumor spheroids grown in confrontation culture, which, however, did not reach statistical significance.

\section{Inhibition of Tumor-Induced Angiogenesis in Confrontation Cultures of Embryoid Bodies and Multicellular Tumor Spheroids by the Free Radical Scavenger Vitamin $E$}

The elevation in intracellular ROS observed in confrontation cultures may be a prerequisite for the progression of tumor-induced angiogenesis. In this respect it has been previously shown that VEGF is up-regulated by ROS (Duyndam et al, 2001). Furthermore, ROS are utilized in VEGF-mediated signal transduction cascades (Colavitti et al, 2002). To evaluate the role of ROS in tumor-induced angiogenesis in confrontation

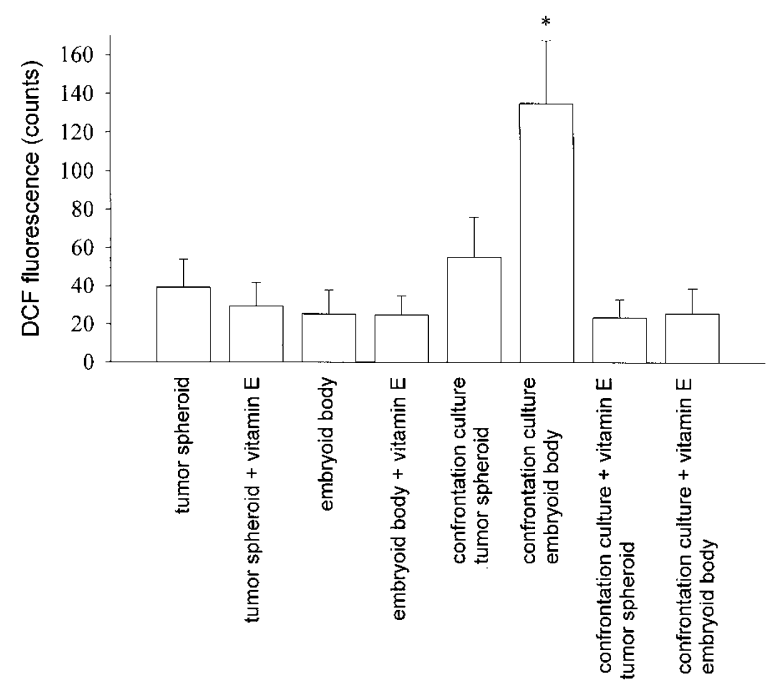

Figure 4.

Elevation of the intracellular redox state upon cultivation of embryoid bodies and multicellular tumor spheroids in confrontation culture. Intracellular reactive oxygen species (ROS) levels were investigated by use of the redoxsensitive dye $\mathrm{H}_{2}$ DCF-DA. Note that confrontation culture resulted in an elevation of intracellular ROS levels, which was most prominent in the embryoid body region of the confrontation culture. cultures of embryoid bodies and multicellular tumor spheroids, cultures were preincubated for 7 days with the free radical scavenger vitamin $\mathrm{E}(100 \mu \mathrm{M})$, and the invasion of endothelial cells was investigated by antiplatelet-endothelial cell adhesion molecule 1 (antiPECAM-1) immunohistochemistry (Fig. 5) $(n=15$ confrontation cultures). It was clear that an invasion of endothelial cells forming capillary-like structures occurred within 4 to 5 days in tumor spheroids grown in untreated confrontation culture. In contrast, cultivation in the presence of vitamin $E$ totally prevented the penetration of endothelial PECAM-1-positive cells into the tumor tissue, which clearly indicates that an elevation of the intracellular redox state is a prerequisite for tumor-induced angiogenesis in confrontation cultures.

\section{Antiangiogenic Activity of Plant Ingredients Used in Traditional Chinese Herbal Medicine}

Ingredients of green tea (eg, catechins) have been previously demonstrated to inhibit angiogenesis and tumor growth (Lamy et al, 2002; Singh et al, 2002). Furthermore, several plant ingredients used in traditional Chinese herbal medicine have been reported to act as free radical scavengers, which may be related to the antiangiogenic activity of the compounds. In the present study, embryoid bodies were incubated from Day 3 to Day 8 of cell culture with baicalein, acteoside, epicatechin, and berberine in different concentrations. Subsequently, embryoid bodies were fixed and stained with an antibody directed against PECAM-1, which specifically stains endothelial cells (Fig. 6, A and B). All the applied agents significantly reduced the area of capillary-like cell structures in a dosedependent manner $(n=3)$, indicating antiangiogenic potency of the herbal ingredients.

In further experiments the effects of plant ingredients on tumor-induced angiogenesis were investigated in confrontation cultures (Fig. 7, A to E). For this purpose confrontation cultures were incubated for 7 days with acteoside $(100 \mu \mathrm{M})$, baicalein $(10 \mu \mathrm{M})$, berberine $(10 \mu \mathrm{M})$, or epicatechin $(30 \mu \mathrm{M})$. It was clear that all applied agents significantly inhibited the penetration of endothelial cells into the tumor tissue, indicating their antiangiogenic activity $(n=15$ confrontation cultures for each substance tested).

\section{Effects of Plant Ingredients Used in Traditional Chinese Herbal Medicine on the Expression of MMPs and Intracellular ROS Levels in Embryoid Bodies}

The antiangiogenic activity of green tea ingredients may be related to an inhibition of MMP expression. It has been recently shown that green tea catechins inhibited MMP expression (Demeule et al, 2000). In the present study, incubation of embryoid bodies for 4 days with acteoside $(100 \mu \mathrm{M})$, baicalein $(10 \mu \mathrm{M})$, berberine $(10 \mu \mathrm{M})$, or epicatechin $(30 \mu \mathrm{M})$ significantly down-regulated the expression of MMP-1 (to $32 \pm$ $8 \%, 45 \pm 8 \%, 46 \pm 24 \%$, and $26 \pm 17 \%$, respectively) and the expression of MMP-2 (to $25 \pm 11 \%, 31 \pm$ $21 \%, 32 \pm 7 \%, 28 \pm 7 \%$, respectively) $(n=3)$ 

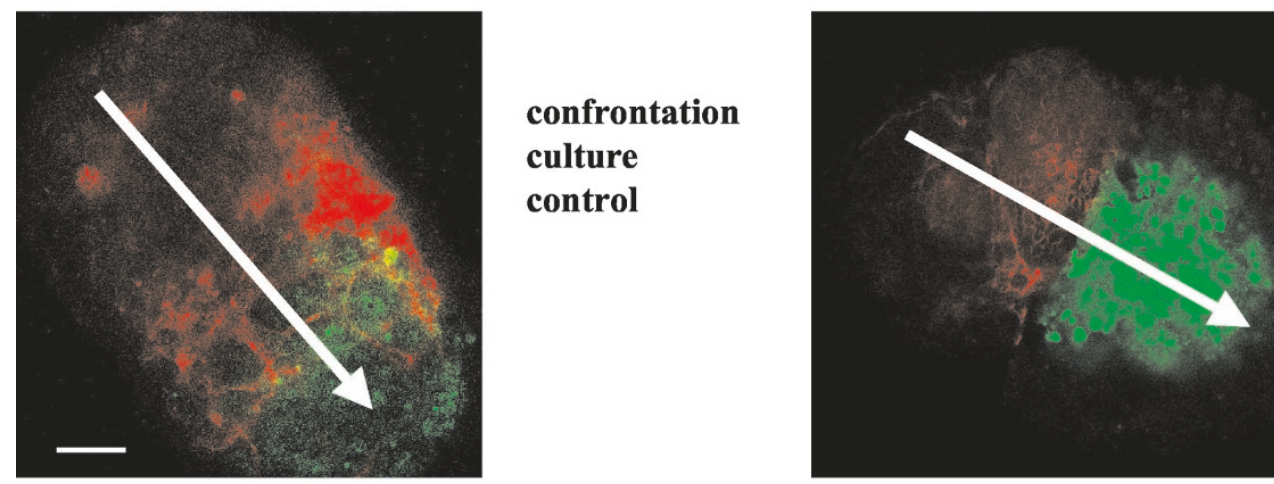

\section{confrontation culture} culture control
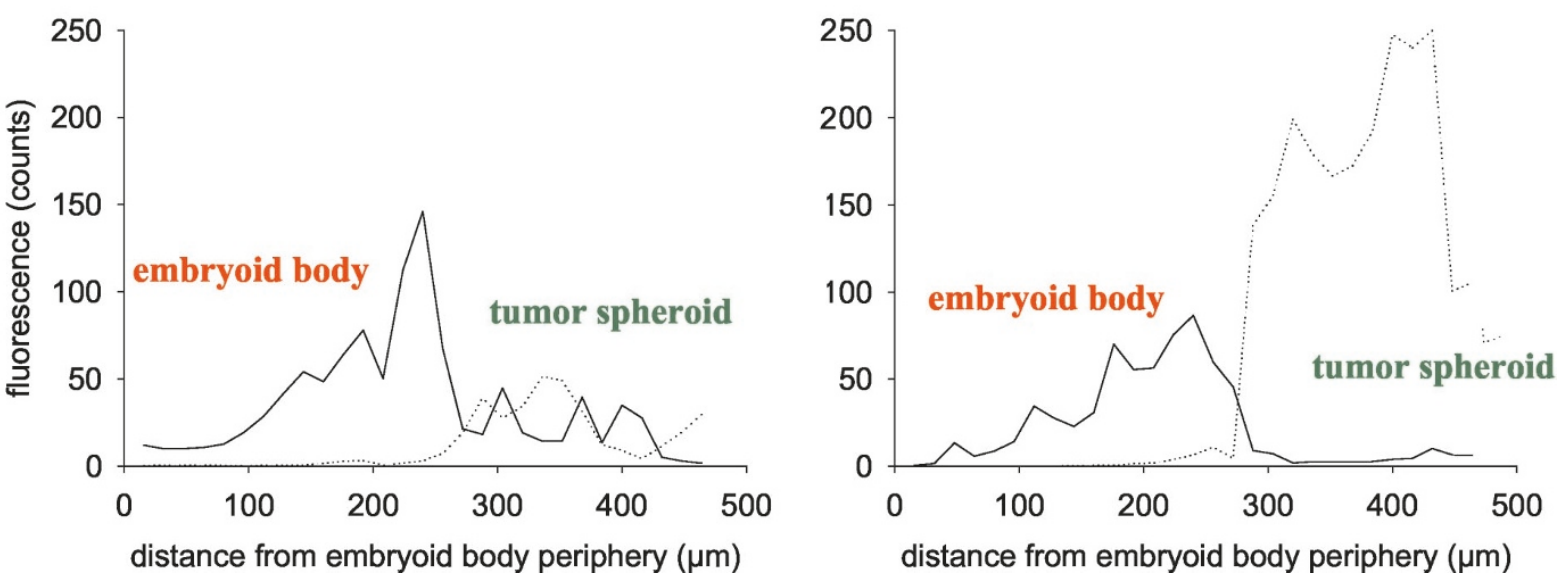

Figure 5 .

Effect of the free radical scavenger vitamin $\mathrm{E}$ on tumor-induced angiogenesis in confrontation cultures consisting of embryoid bodies and multicellular tumor spheroids (5 days of confrontation culture). Endothelial cells were visualized by anti-platelet-endothelial cell adhesion molecule 1 (anti-PECAM-1) immunohistochemistry (red color). The tissue of multicellular tumor spheroids was visualized by labeling with the long-term cell tracker dye 5-chloromethylfluorescein diacetate (green color). Upper panel, Representative confrontation cultures that either remained untreated (A) or were treated for 7 days with vitamin $\mathrm{E}$ (100 $\mu \mathrm{m}$ ) (B). The bar represents $100 \mu \mathrm{m}$. It is clear that in the control sample, PECAM-1-positive capillary-like structures invaded the tumor spheroid, whereas no invasion occurred in the vitamin E-treated sample. Lower panel, Histograms of PECAM-1 fluorescence (solid line) and CMF fluorescence (dotted line) along the arrow in the images. Data are presented as fluorescence counts in relation to the distance from the embryoid body periphery.

(untreated control set to $100 \%$ ), which indicates that the antiangiogenic effect of the applied compound may at least in part be mediated through the downregulation of MMP expression (Fig. 8). Interestingly, no down-regulation of MMP-9 was observed $(n=3)$, which may, however, be related to the fact that MMP-9 is expressed at only low levels in embryoid bodies (Fig. 1).

The observed inhibition of MMP expression by plant ingredients may be a result of a decrease in the intracellular ROS levels because MMP expression is regulated by the intracellular redox state (Belkhiri et al, 1997; Brenneisen et al, 1997; Yoon et al, 2002). To investigate this, we labeled embryoid bodies labeled with the redox sensitive dye $\mathrm{H}_{2}$ DCF-DA and we monitored the intracellular ROS levels. It was evident that baicalein, acteoside, epicatechin, and berberine significantly reduced the time kinetics of oxidation of $\mathrm{H}_{2}$ DCF to the highly fluorescent oxidation product DCF, indicating that the compounds were acting as scavengers of intracellular free radicals $(n=3)$ (Fig. 9).

\section{Discussion}

Antiangiogenic therapy is currently one of the most promising and efficient therapies against cancer. This has initiated intensive research for novel antiangiogenic agents that exert specific effects on endothelial cell migration and/or proliferation as well as for innovative in vitro models to study the efficacy of antiangiogenic compounds. We recently introduced a novel in vitro system for routine evaluation of antiangiogenic agents (ie, ES cell-derived embryoid bodies) (Wartenberg et al, 1998) as well as confrontation cultures consisting of embryoid bodies and multicellular tumor spheroids (Wartenberg et al, 2001a). Our previous data and the data of the present study clearly demonstrate that the applied in vitro models are excellently suited for antiangiogenesis research.

The present data show that confrontation culture of multicellular tumor spheroids and embryoid bodies results in transient elevations of MMP-1, MMP-2, and MMP-9, which are expressed in a distinctly regulated time frame during confrontation culture. MMPs are 

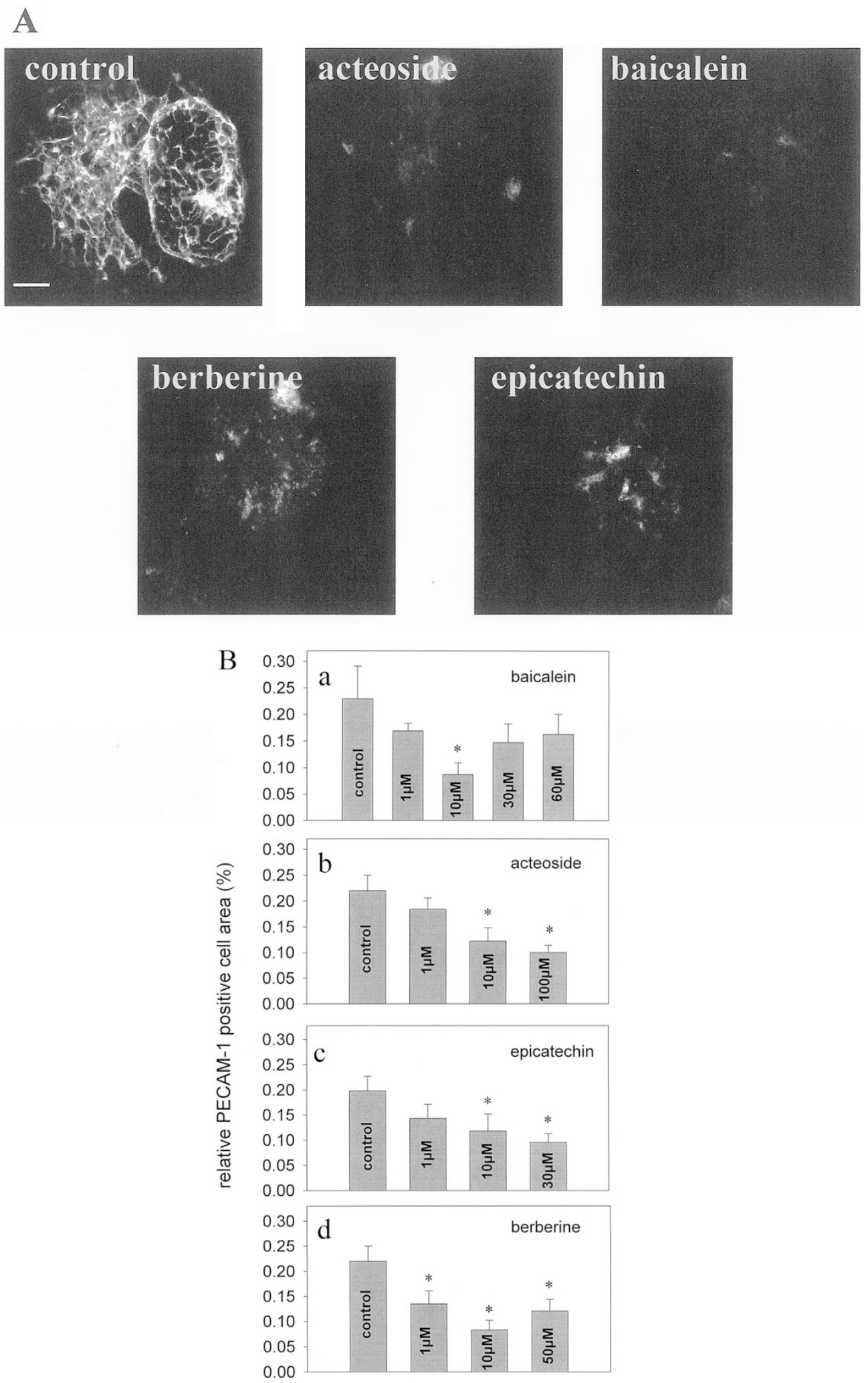

\section{Figure 6.}

Effects of plant ingredients used in traditional Chinese herbal medicine on vasculogenesis and angiogenesis of embryoid bodies. Embryoid bodies were treated for 4 days (Days 4 to 8 of cell culture) with either baicalein, acteoside, epicatechin, or berberine in concentrations as indicated. Subsequently, they were fixed, anti-PECAM-1 immunohistochemistry was performed, and the PECAM-1-positive area was evaluated in optical sections of 100- $\mu \mathrm{M}$ thickness. A, Representative embryoid bodies that either remained untreated (control) or were treated with acteoside (100 $\mu \mathrm{M})$, baicalein (10 $\mu \mathrm{M})$, berberine $(10 \mu \mathrm{M})$, or epicatechin $(30 \mu \mathrm{M})$. The bar represents $100 \mu \mathrm{m}$. B, Quantitative evaluation of PECAM-1-positive cell areas in embryoid bodies treated with plant ingredients in concentrations as indicated. a, baicalein; b, acteoside; c, epicatechin; d, berberine. 
A

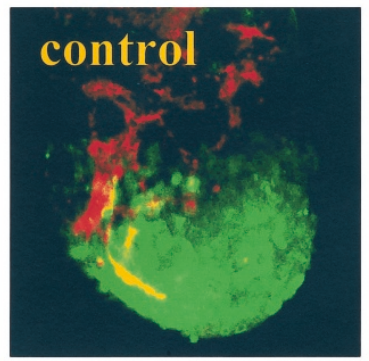

B

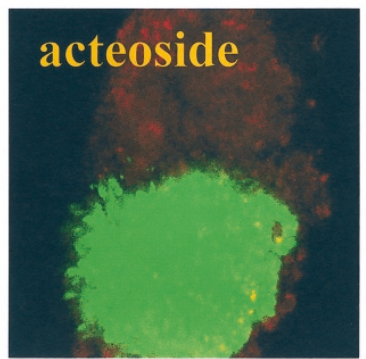

C epicatechin
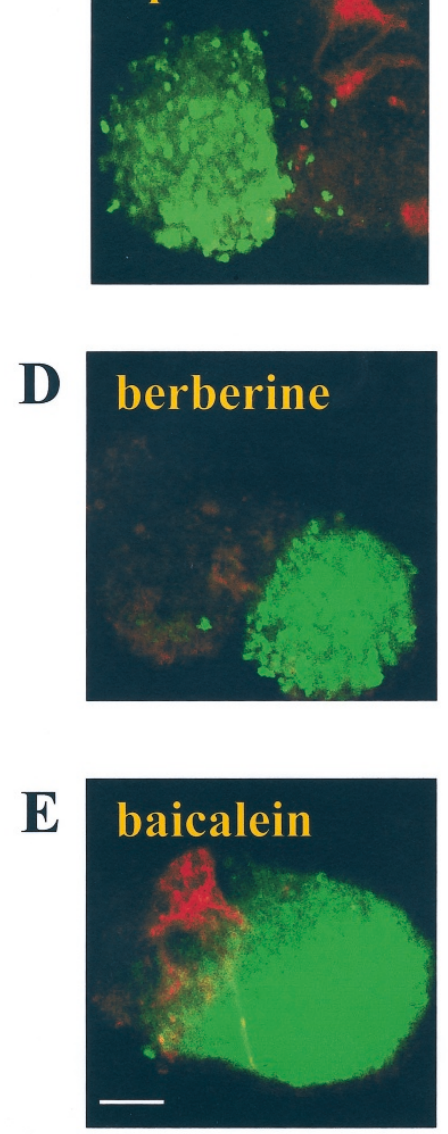
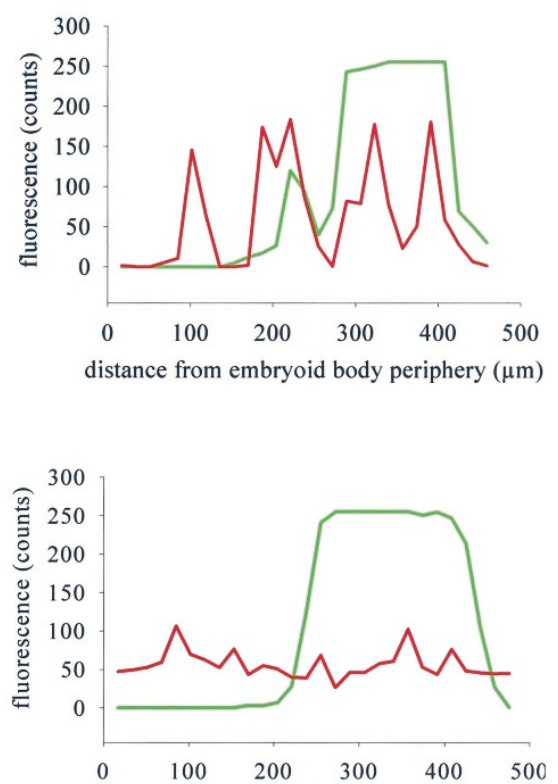

distance from embryoid body periphery $(\mu \mathrm{m}$

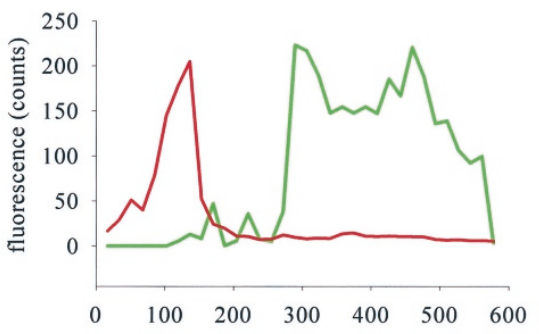

distance from embryoid body periphery $(\mu \mathrm{m})$
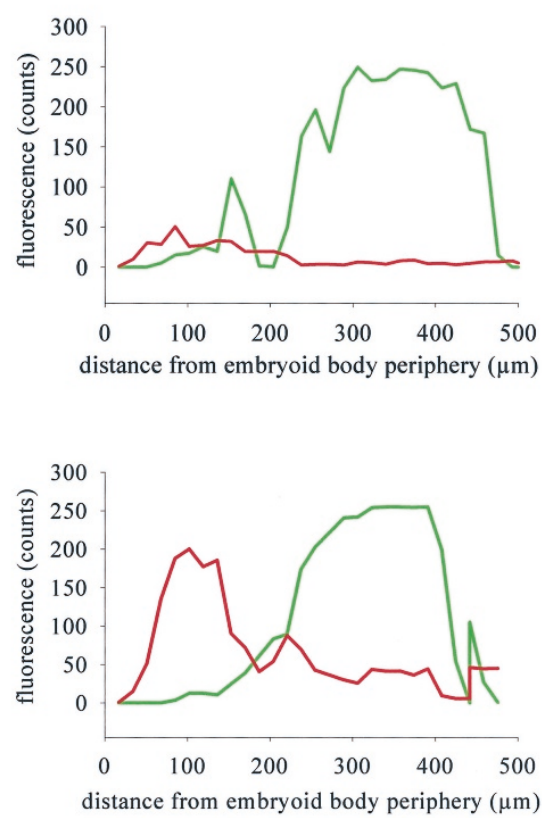

Figure 7.

Effects of plant ingredients used in traditional Chinese herbal medicine on tumor-induced angiogenesis in confrontation cultures. Confrontation cultures were treated for 7 days with either acteoside, epicatechin, berberine, or baicalein in concentrations as indicated. Subsequently, they were fixed, anti-PECAM-1 immunohistochemistry was performed, and the PECAM-1-positive area was evaluated in optical sections of 100- $\mu$ m thickness. Left panels, Representative confrontation cultures that either remained untreated (control) $(A)$ or were treated with acteoside $(100 \mu \mathrm{M})(B)$, epicatechin $(30 \mu \mathrm{M})(\mathrm{C})$, berberine $(10 \mu \mathrm{M})(\mathrm{D})$, or baicalein $(10 \mu \mathrm{M})(\mathrm{E})$. Right panels, Histograms of PECAM-1 fluorescence (red line) and CMF fluorescence (green line). Data are presented as fluorescence counts in relation to the distance from the embryoid body periphery. The bar represents $100 \mu \mathrm{m}$. Note that in confrontation cultures treated with plant ingredients, invasion of endothelial cell structures was nearly completely abolished. 


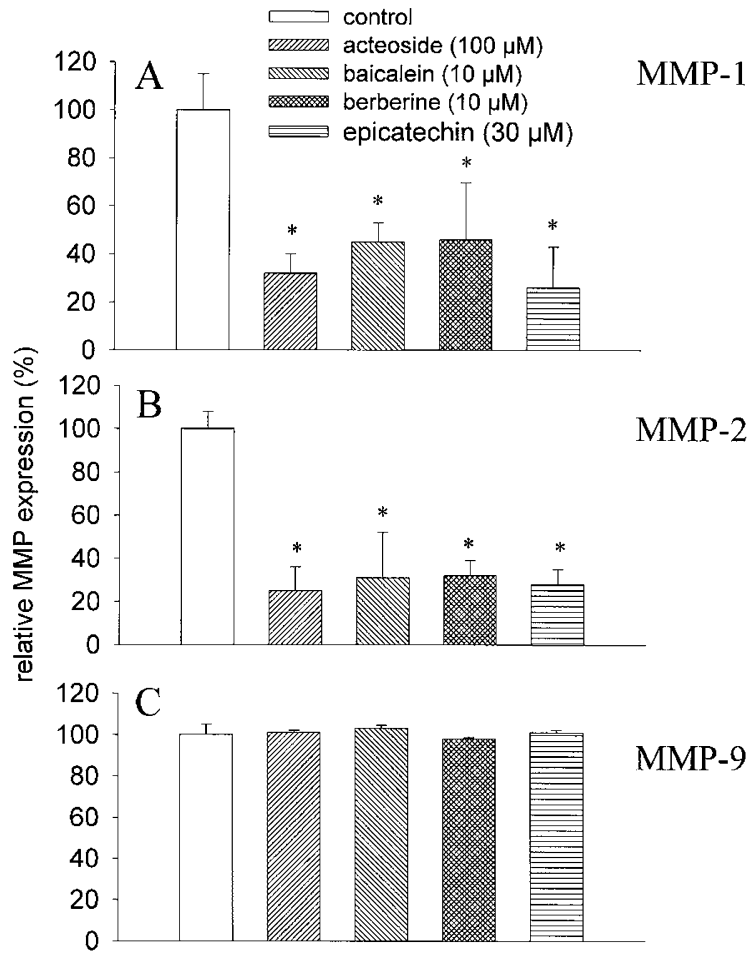

Figure 8.

Effects of plant ingredients used in traditional Chinese herbal medicine on the expression of (A) MMP-1, (B) MMP-2, and (C) MMP-9 in embryoid bodies Embryoid bodies were treated for 48 hours (Days 6 to 8 of cell culture) with acteoside $(100 \mu \mathrm{M})$, baicalein $(10 \mu \mathrm{M})$, berberine $(10 \mu \mathrm{M})$, or epicatechin $(30$ $\mu \mathrm{M})$. Note that all applied plant ingredients significantly down-regulated MMP-1 and MMP-2 expression, whereas no down-regulation of MMP-9 expression was observed.

well known to be necessary for tumor-induced angiogenesis because endothelial cell invasion requires the degradation of the extracellular matrix in the tumor tissue (Johansson et al, 2000). MMP expression has been previously shown to be regulated by the intracellular redox state. This has been evidenced in particular for MMP-1, MMP-2, and MMP-9. Exogenous addition of $\mathrm{H}_{2} \mathrm{O}_{2}$ increases the steady-state mRNA levels of collagenase/MMP-1 in human dermal fibroblasts (Brenneisen et al, 1997) and activates MMP-2 in endothelial cells (Belkhiri et al, 1997). Activation of MMP-2 has been likewise demonstrated to occur in endothelial cells through lysophosphatidylcholineinduced ROS (Inoue et al, 2001), and it was conclusively shown that the signaling cascade resulting in $\mathrm{H}_{2} \mathrm{O}_{2}$-induced proMMP-2 activation occurs through the receptor tyrosine kinase/phosphatidylinositol 3-kinase/NF-kappa B pathway (Yoon et al, 2002). Activation of MMP-9 has been demonstrated to occur via ROS upon IL-1 treatment of vascular smooth muscle cells (Gurjar et al, 2001). It has been recently shown that MMP-9 triggers the angiogenic switch during carcinogenesis (Bergers et al, 2000).

The data of the present study demonstrate that MMP expression may likewise be regulated by the intracellular redox state in confrontation cultures of multicellular tumor spheroids and embryoid bodies because confrontation culture resulted in an elevation

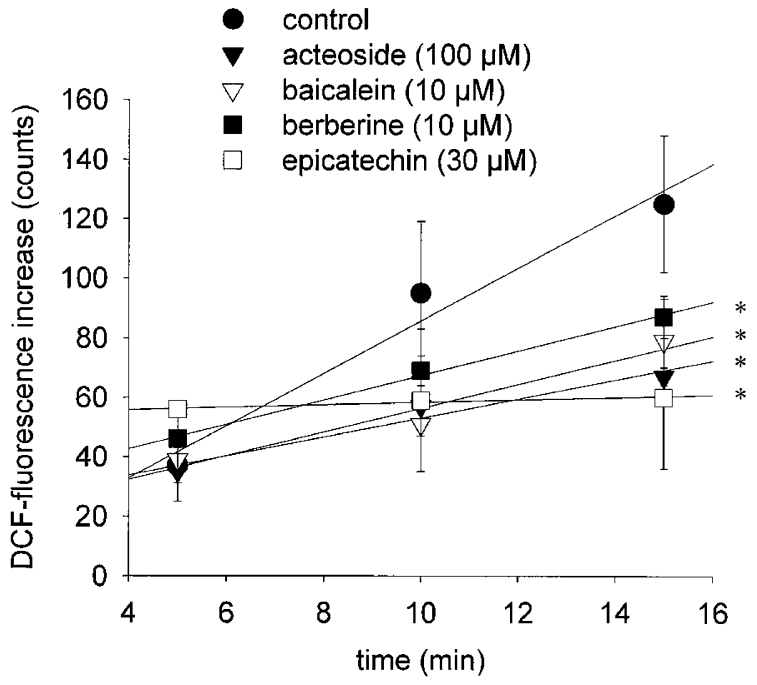

Figure 9.

Effects of plant ingredients used in traditional Chinese herbal medicine on intracellular ROS levels in embryoid bodies. Embryoid bodies were incubated for 24 hours with acteoside $(100 \mu \mathrm{M})$, baicalein $(10 \mu \mathrm{M})$, berberine $(10 \mu \mathrm{M})$, or epicatechin $(30 \mu \mathrm{m})$. Subsequently they were stained with the redox-sensitive dye $\mathrm{H}_{2}$ DCF-DA, and fluorescent DCF was monitored after 5,10 , and 15 minutes. Note that the time course of $\mathrm{H}_{2} \mathrm{DCF}$ oxidation was significantly reduced upon treatment with the compounds, indicating that they are acting as free radical scavengers.

of intracellular ROS levels, presumably through the activity of an NADPH oxidase, which we have previously shown to be expressed in multicellular tumor spheroids of the DU-145 prostate cancer cell line (Wartenberg et al, 2001b) and in ES cell-derived embryoid bodies (Sauer et al, 2000b). Furthermore, the observed increase in MMP expression in the tissue of multicellular tumor spheroids and embryoid bodies upon confrontation culture was completely abrogated after incubation with the free radical scavenger vitamin E. Consequently vitamin $\mathrm{E}$ treatment totally abolished tumor-induced angiogenesis in confrontation cultures (ie, the invasion of blood vessel-like structures into the tumor tissue).

We investigate the plant ingredients acteoside, baicalein, berberine, and epicatechin for their antiangiogenic capacity in the embryoid body in vitro model. We demonstrated that all applied agents severely inhibited angiogenesis and capillary growth in the embryoid body model, indicating that these agents may be suitable in antiangiogenic treatment of cancer. Interestingly, all applied agents significantly reduced the intracellular redox state, suggesting that they are acting as free radical scavengers. Consequently, plant ingredients significantly down-regulated MMP expression, indicating that the antiangiogenic effect of the compounds may be transduced through an inhibition of redox-regulated MMP expression. The antioxidative capacity of plant ingredients used in traditional Chinese medicine has been previously demonstrated for baicalein (Hamada et al, 1993; Shao et al, 2002), epicatechin (Harada et al, 1999; Terao, 1999; Zhang et al, 2000), berberine (Choi et al, 2001), and acteoside (Wong et al, 2001a). Inhibition of MMP-2 and MMP-9 
expression has been demonstrated for various catechins (Demeule et al, 2000), and it has been discussed whether the ability of these compounds to reduce MMP expression may be exploited in angioprevention (Tosetti et al, 2002). It has been recently demonstrated that epigallocatechin-3-gallate inhibits angiogenic differentiation of human endothelial cells (Singh et al, 2002). Baicalein, berberine, and acteoside have so far not been demonstrated to inhibit MMP expression; this should, however, be expected because we and others have demonstrated that these compounds exert pronounced antioxidative capacity.

Several molecular mediators of the inflammatory process and related signaling pathways are involved in tumorigenesis and angiogenesis. As a general mechanism, oxidative stress is a common hallmark of inflammation and the tumoral phenotype. Tumor growth produces large amounts of ROS (Szatrowski and Nathan, 1991; Wartenberg et al, 2000), which can induce MMP expression and can attract tumorinfiltrating leukocytes to induce the angiogenic response (Shono et al, 1996). ROS have been shown to increase VEGF expression, thereby promoting endothelial cell growth. Furthermore, ROS are well known to activate and modulate signal transduction cascades including signaling pathways involved in angiogenesis (eg, VEGF-induced endothelial cell signaling) (Colavitti et al, 2002). Recently, it has been demonstrated that ROS generated by Nox1, which is a nonphagocytic NADPH oxidase, triggers the angiogenic switch (Arbiser et al, 2002). Overexpression of Nox1 increased the vascularity of tumors and induced molecular markers of angiogenesis including VEGF and MMPs (Arbiser et al, 2002). Inhibition of MMPs, required for endothelial cell invasion, has been previously demonstrated to be a common target of active chemopreventive agents (Garbisa et al, 1999). It therefore seems conceivable to exploit the antioxidative capacity of plant ingredients that have been successfully applied during many centuries in traditional Chinese medicine to achieve angioprevention in cancer.

\section{Materials and Methods}

\section{Materials}

Baicalein was isolated and purified from Scutellaria baicalensis Georgi (Huangqin) (Chen et al, 2000). In brief, the ground roots of Huangqin were extracted with acetone, followed by evaporation. The acetone extract was then dissolved in methanol. Bacalein as a yellow crystal was isolated and redissolved in methanol followed by recrystallization at $-20^{\circ} \mathrm{C}$.

To isolate and purify $(-)$ epicatechin, jasmine tea leaves were used (Huang et al, 1998). The tea leaves were soaked in hot distilled water, and the filtered infusion was then extracted with chloroform to remove caffeine and pigments. The remaining aqueous phase was extracted with ethyl acetate, and the ethyl acetate phase containing epicatechins was collected, followed by evaporation. The resulting crude epicatechin extracts were dissolved in distilled water and freeze- dried overnight. Separation of (-) epicatechin from the crude extracts was performed by Shimazu LC-10AD HPLC (Toko, Japan) as previously described by Zhang et al (1997). Acteoside was isolated and purified from L. purpurascens (Wong et al, 2001b). Dried leaves of $L$. purpurascens were extracted with ethanol, followed by evaporation. After the evaporated extract was dissolved in water, it was fractionated to yield two fractions, $\mathrm{A} 1$ and $\mathrm{A} 2$. The A2 fraction, which contained crude glycosides, was chromatographed on a silica gel with $\mathrm{CHCl}_{3}-\mathrm{MeOH}-\mathrm{H}_{2} \mathrm{O}$ of decreasing $\mathrm{CHCl}_{3}$ content. The eluate with $\mathrm{CHCl}_{3}-\mathrm{MeOH}-\mathrm{H}_{2} \mathrm{O}(40: 10: 1)$ was concentrated to give residue. This residue was purified by LC on Si 60 (Lobar, 40-63 $\mu \mathrm{m}$; Merck, Darmstadt, Germany) with $\mathrm{CHCl}_{3}-\mathrm{MeOH}-\mathrm{H}_{2} \mathrm{O}$ (7:3:0.5) and Sephadex $\mathrm{LH}-20$ with $\mathrm{EtOH}$ to yield acteoside. The purity of baicalein, (-) epicatechin, and acteoside extracted by us was greater than $99 \%$ by HPLC analysis. Berberine, an isoquinoline alkaloid derived from Huanglian and many other plants, such as the Berberidaceae family, was purchased from Sigma (St. Louis, Missouri).

\section{Spinner-Culture Technique for Cultivation of Embryoid Bodies}

The ES cell line CCE was grown on mitotically inactivated feeder layers of primary murine embryonic fibroblasts for a maximum of eight passages in Iscove's medium (Gibco, Life Technologies, Gaithersburg, Maryland). Medium was supplemented with $20 \%$ heat-inactivated $\left(56^{\circ} \mathrm{C}, 30\right.$ minutes) FCS (BoehringerMannheim, Mannheim, Germany), 2 mм Glutamax (Gibco), $100 \mu \mathrm{M} \beta$-mercaptoethanol (Sigma, Deisenhofen, Germany), 1\% MEM nonessential amino acids stock solution (Gibco), and $100 \mathrm{IU} / \mathrm{ml}$ penicillin and $100 \mu \mathrm{g} / \mathrm{ml}$ streptomycin (both Gibco) in a humidified environment containing $5 \% \mathrm{CO}_{2}$ at $37^{\circ} \mathrm{C}$ and passaged every 2 to 3 days. At Day 0 of differentiation, adherent cells were enzymatically dissociated using $0.2 \%$ trypsin and $0.05 \%$ EDTA in PBS (Gibco) and seeded at a density of $1 \cdot 10^{7}$ cells $\mathrm{ml}^{-1}$ in $250 \mathrm{ml}$ of siliconized spinner flasks (Integra Biosciences, Fernwald, Germany) containing $100 \mathrm{ml}$ of Iscove's medium supplemented with the same additive as described above. After 24 hours, $150 \mathrm{ml}$ of medium was added to give a final volume of $250 \mathrm{ml}$. The spinner flask medium was stirred at $20 \mathrm{rpm}$ using a stirrer system (Integra Biosciences), and $150 \mathrm{ml}$ of cell culture medium was exchanged every day.

\section{Spinner-Culture Technique for Cultivation of Multicellular Tumor Spheroids}

The human prostate cancer cell line DU-145 was used throughout the study. The cell line was grown routinely in $5 \% \mathrm{CO}_{2}$ /humidified air at $37^{\circ} \mathrm{C}$ with Ham's $\mathrm{F} 10$ medium (Gibco) supplemented with 10\% FCS (Boehringer), 2 mм glutamine, $100 \mu \mathrm{M} \beta$-mercaptoethanol, 2 $\mathrm{mm} \mathrm{MEM}$, and $100 \mathrm{lU} / \mathrm{ml}$ penicillin and $100 \mu \mathrm{g} / \mathrm{ml}$ streptomycin (Gibco). Spheroids were grown from single cells. Cell monolayers were trypsinized with 
$0.2 \%$ trypsin/0.05\% EDTA (Gibco) and seeded in 250-ml spinner flasks as described previously (Wartenberg et al, 2001a). For our experiments, tumor spheroids with mean diameters of $440 \pm 80 \mu \mathrm{m}$ were used. Central necrosis was obvious in these tumor spheroids.

\section{Generation of Confrontation Cultures}

For the generation of confrontation cultures, multicellular tumor spheroids and embryoid bodies were removed from spinner flasks. One embryoid body (5 to 6 days old) together with one tumor spheroid (18 to 30 days old) was added to a 40- $\mu$ l drop of Iscove's cell culture medium placed onto the lid of a $10-\mathrm{cm}$ Petri culture dish. After adding 20 to 30 drops to the lid of the Petri dish, the lid was turned around and placed on the Petri dish, which was filled with $10 \mathrm{ml}$ of sterile PBS. Within 24 to 48 hours, embryoid bodies and tumor spheroids closely attached within the hanging drops and were subsequently transferred to $10-\mathrm{cm}$ bacteriologic Petri dishes filled with $10 \mathrm{ml}$ of Iscove's cell culture medium. Confrontation cultures were used for the experiments at the indicated times.

\section{Confocal Laser Scanning Microscopy}

Fluorescence recordings were performed by means of a confocal laser scanning setup (LSM 410; Zeiss, Jena, Germany) connected to an inverted microscope (Axiovert 135; Zeiss). The confocal setup was equipped with a 5-mW helium/neon laser, with single excitation of $633 \mathrm{~nm}$ (excitation of Cy5), a $0.5-\mathrm{mW}$ helium neon laser, with single excitation of $543 \mathrm{~nm}$ (excitation of Cy3), and an argon laser, with single excitation of $488 \mathrm{~nm}$ (excitation of CMF). Emission was recorded using the longpass filter sets LP570, RG665, and LP515, respectively. A $16 \times$ numerical aperture 0.5 , oil immersion-corrected objective (Neofluar; Zeiss) was used.

\section{Long-Term Labeling of Multicellular Tumor Spheroids}

To discriminate tumor spheroids grown in confrontation culture from embryoid bodies, tumor spheroids were labeled with the long-term cell tracker dye 5-chloromethylfluorescein diacetate (CMFDA) (Molecular Probes, Eugene, Oregon). In brief, tumor spheroids were incubated for 60 minutes in F10 cell culture medium that contained $10 \mu \mathrm{M}$ CMFDA (stock solution $10 \mathrm{~mm}$, dissolved in dimethyl sulfoxide). Subsequently, spheroids were washed and incubated for a further 24 hours in bacteriologic Petri dishes. The penetration of CMFDA into the tumor tissue was assessed in diffusion experiments as described previously (Wartenberg and Acker, 1995). In brief, tumor spheroids were incubated with the dye, and the CMFDA fluorescence was determined in selected regions of interest $(600$ $\mu \mathrm{m}^{2}, 40 \times 40$ pixel) after 30 minutes by performing optical sections with a thickness of $5 \mu \mathrm{m}$ from the periphery toward the center of the tumor spheroids. The diffusion coefficient $D$ was then calculated according to the Einstein-Smoluchovski equation: $\mathrm{D}=$ $x^{2} / 2 t$, where $D$ is the diffusion coefficient, $x$ is the maximal diffusion distance of CMFDA from the tumor spheroid periphery, and $t$ is the diffusion time. From this equation the diffusion coefficient $D$ for CMFDA in the tissue of multicellular tumor spheroids was calculated as $3.4 \cdot 10^{-8} \mathrm{~cm}^{2} \mathrm{~s}^{-1}$. Accordingly, a penetration depth of $156 \mu \mathrm{m}$ for CMFDA was achieved within 1 hour of incubation. Stable CMF fluorescence was observed for more than 5 days of tumor spheroid culture. Fluorescence excitation was performed by the 488-nm line of an argon-ion laser of the confocal setup. Emission was recorded using a longpass LP515-nm filter set.

\section{Immunohistochemistry}

Immunohistochemistry was performed with whole mount embryoid bodies, tumor spheroids, and confrontation cultures. As primary antibodies the monoclonal anti-mouse PECAM-1 (CD31) (Endogen, Woburn, Massachusetts) (concentration of $5 \mu \mathrm{g} / \mathrm{ml}$ ), the mouse monoclonal anti-MMP-1 (dilution 1:100) and anti-MMP-9 (dilution 1:100) (Oncogene Research Products, Boston, Massachusetts), and the polyclonal rabbit anti-MMP-2 (dilution 1:100) (Chemicon, Temecula, California) were used. For PECAM-1 staining the respective tissues were fixed in ice-cold methanol/ acetone (7:3) for 60 minutes at $-20^{\circ} \mathrm{C}$ and washed with PBS containing $0.1 \%$ Triton $\mathrm{X}-100$ (PBST) (Sig$\mathrm{ma})$. For MMP staining the tissues were fixed for 1 hour at $4^{\circ} \mathrm{C}$ in $4 \%$ formaldehyde in PBS. Blocking against unspecific binding was performed for 60 minutes with $10 \%$ fat-free milk powder (Heirler, Radolfzell, Germany) dissolved in PBS. The tissues were subsequently incubated for 90 minutes (for MMP staining overnight at $8^{\circ} \mathrm{C}$ ) at room temperature with primary antibodies dissolved in PBS supplemented with $10 \%$ milk powder. The tissues were thereafter washed three times with PBST (0.01\% Triton) and reincubated with either a Cy5-conjugated rabbit anti-Syrian hamster IgG $(\mathrm{H}+\mathrm{L})$ (PECAM-1), a Cy5-conjugated goat anti-mouse $\lg \mathrm{G}(\mathrm{H}+\mathrm{L})$ (MMP-1 and MMP-9), or a Cy3-conjugated goat anti-rabbit IgG (MMP-2) (all from Dianova, Hamburg, Germany) at a concentration of $3.8 \mu \mathrm{g} / \mathrm{ml}$ in PBS containing $10 \%$ milk powder. After washing three times in PBST (0.01\% Triton), the tissues were stored in PBS until inspection. For the excitation of the Cy5 fluorochrome, the 633-nm band of a helium/neon laser of the confocal setup was used. Emission was recorded using a 655-nm longpass filter set. The Cy3 fluorochrome was excited by the 543-nm band of a helium/neon laser, and emission was recorded using a 570-nm longpass filter set. The pinhole settings of the confocal setup were adjusted to give a full width half maximum of $9 \mu \mathrm{m}$. Fluorescence was recorded in a depth of 80 to $120 \mu \mathrm{m}$ in the tissue, and the fluorescence values in the respective optical section were evaluated by the image analysis software of the confocal setup.

The immunofluorescence values obtained in the experiments were corrected for fluorescence arising from unspecific binding of the primary and secondary 
antibodies within the tissue. To assess the magnitude of unspecific fluorescence, the tissues were incubated with nonsense antibodies directed against antigens that are not expressed in the respective tissue, such as an antibody against prostate-specific acid phosphatase (Sigma) used in embryoid bodies and the erythroid cell-specific antibody TER-119 (PharMingen, Hamburg, Germany) used in tumor spheroids. Labeling with these primary antibodies and a Cy-5-labeled secondary antibody resulted in a weak unspecific fluorescence, which was subtracted from the fluorescence values achieved in the immunofluorescence experiments with PECAM-1 and MMPs.

\section{Measurement of ROS Generation}

Intracellular ROS levels were measured using the fluorescent dye $\mathrm{H}_{2}$ DCF-DA (Molecular Probes), which is a nonpolar compound that is converted into a nonfluorescent polar derivative $\left(\mathrm{H}_{2} \mathrm{DCF}\right)$ by cellular esterases after incorporation into cells. $\mathrm{H}_{2} \mathrm{DCF}$ is membrane impermeable and is rapidly oxidized to the highly fluorescent DCF in the presence of intracellular ROS. For the experiments, multicellular tumor spheroids were incubated in $\mathrm{E} 1$ medium (containing in $\mathrm{mm}$ : $\mathrm{NaCl} 135, \mathrm{KCl} 5.4, \mathrm{CaCl}_{2} 1.8, \mathrm{MgCl}_{2}$ 1, glucose 10, HEPES 10 [pH 7.4 at $\left.23^{\circ} \mathrm{C}\right]$ ), and $20 \mu \mathrm{M} \mathrm{H} \mathrm{H}_{2}$ DCF-DA dissolved in dimethyl sulfoxide was added. After 20 minutes intracellular DCF fluorescence (corrected for background fluorescence) was evaluated in $3600 \mu \mathrm{m}^{2}$ regions of interest using an overlay mask unless otherwise indicated. For fluorescence excitation, the 488-nm band of the argon ion laser of the confocal setup was used. Emission was recorded using a longpass LP515-nm filter set.

\section{Statistical Analysis}

Data are given as mean values $\pm \mathrm{SD}$, with $n$ denoting the number of experiments unless otherwise indicated. In each experiment at least 15 embryoid bodies or confrontation culture objects were analyzed. Student's $t$ test for unpaired data was applied as appropriate. A value of $p<0.05$ was considered significant.

\section{References}

Abid MR, Kachra Z, Spokes KC, and Aird WC (2000). NADPH oxidase activity is required for endothelial cell proliferation and migration. FEBS Lett 486:252-256.

Angkeow P, Deshpande SS, Qi B, Liu YX, Park YC, Jeon BH, Ozaki M, and Irani K (2002). Redox factor-1: An extra-nuclear role in the regulation of endothelial oxidative stress and apoptosis. Cell Death Differ 9:717-725.

Arbiser JL, Petros J, Klafter R, Govindajaran B, McLaughlin ER, Brown LF, Cohen C, Moses M, Kilroy S, Arnold RS, and Lambeth JD (2002). Reactive oxygen generated by Nox1 triggers the angiogenic switch. Proc Natl Acad Sci USA 99:715-720.

Belkhiri A, Richards C, Whaley M, McQueen SA, and Orr FW (1997). Increased expression of activated matrix metalloproteinase-2 by human endothelial cells after sublethal $\mathrm{H} 2 \mathrm{O} 2$ exposure. Lab Invest 77:533-539.
Bergers G, Brekken R, McMahon G, Vu TH, Itoh T, Tamaki K, Tanzawa K, Thorpe P, Itohara S, Werb Z, and Hanahan D (2000). Matrix metalloproteinase- 9 triggers the angiogenic switch during carcinogenesis. Nat Cell Biol 2:737-744.

Brenneisen P, Briviba K, Wlaschek M, Wenk J, and Scharffetter-Kochanek K (1997). Hydrogen peroxide (H2O2) increases the steady-state mRNA levels of collagenase/ MMP-1 in human dermal fibroblasts. Free Radic Biol Med 22:515-524.

Chen ZY, Su YL, Bi YR, Tsang SY, and Huang Y (2000). Effect of baicalein and acetone extract of Scutellaria baicalensis on canola oil oxidation. J Am Oil Chem Soc 77:73-78.

Choi DS, Kim SJ, and Jung MY (2001). Inhibitory activity of berberine on DNA strand cleavage induced by hydrogen peroxide and cytochrome c. Biosci Biotechnol Biochem 65:452-455.

Colavitti R, Pani G, Bedogni B, Anzevino R, Borrello S, Waltenberger J, and Galeotti T (2002). Reactive oxygen species as downstream mediators of angiogenic signaling by vascular endothelial growth factor receptor-2/KDR. J Biol Chem 277:3101-3108.

Demeule M, Brossard M, Page M, Gingras D, and Beliveau R (2000). Matrix metalloproteinase inhibition by green tea catechins. Biochim Biophys Acta 1478:51-60.

Duyndam MC, Hulscher TM, Fontijn D, Pinedo HM, and Boven E (2001). Induction of vascular endothelial growth factor expression and hypoxia-inducible factor 1alpha protein by the oxidative stressor arsenite. J Biol Chem 276: 48066-48076.

Folkman J (1971). Tumor angiogenesis: Therapeutic implications. N Engl J Med 285:1182-1186.

Folkman J, Merler E, Abernathy C, and Williams G (1971). Isolation of a tumor factor responsible for angiogenesis. J Exp Med 133:275-288.

Garbisa S, Biggin S, Cavallarin N, Sartor L, Benelli R, and Albini A (1999). Tumor invasion: Molecular shears blunted by green tea. Nat Med 5:1216.

Gurjar MV, Deleon J, Sharma RV, and Bhalla RC (2001). Mechanism of inhibition of matrix metalloproteinase-9 induction by NO in vascular smooth muscle cells. J Appl Physiol 91:1380-1386.

Hamada H, Hiramatsu M, Edamatsu R, and Mori A (1993). Free radical scavenging action of baicalein. Arch Biochem Biophys 306:261-266.

Harada M, Kan Y, Naoki H, Fukui Y, Kageyama N, Nakai M, Miki W, and Kiso Y (1999). Identification of the major antioxidative metabolites in biological fluids of the rat with ingested $(+)$-catechin and (-)-epicatechin. Biosci Biotechnol Biochem 63:973-977.

Heitzer T, Schlinzig T, Krohn K, Meinertz T, and Munzel T (2001). Endothelial dysfunction, oxidative stress, and risk of cardiovascular events in patients with coronary artery disease. Circulation 104:2673-2678.

Huang Y, Zhang A, Lau CW, and Chen ZY (1998). Vasorelaxant effects of purified green tea epicatechin derivatives in rat mesenteric artery. Life Sci 63:275-283.

Inoue N, Takeshita S, Gao D, Ishida T, Kawashima S, Akita H, Tawa R, Sakurai H, and Yokoyama M (2001). Lysophosphatidylcholine increases the secretion of matrix metalloprotein- 
ase 2 through the activation of NADH/NADPH oxidase in cultured aortic endothelial cells. Atherosclerosis 155:45-52.

Johansson N, Ahonen M, and Kahari VM (2000). Matrix metalloproteinases in tumor invasion. Cell Mol Life Sci 57:515.

Lamy S, Gingras D, and Beliveau R (2002). Green tea catechins inhibit vascular endothelial growth factor receptor phosphorylation. Cancer Res 62:381-385.

Sauer H, Gunther J, Hescheler J, and Wartenberg M (2000a). Thalidomide inhibits angiogenesis in embryoid bodies by the generation of hydroxyl radicals. Am J Pathol 156:151-158.

Sauer H, Rahimi G, Hescheler J, and Wartenberg M (2000b). Role of reactive oxygen species and phosphatidylinositol 3-kinase in cardiomyocyte differentiation of embryonic stem cells. FEBS Lett 476:218-223.

Shao ZH, Vanden Hoek TL, Qin Y, Becker LB, Schumacker PT, Li CQ, Dey L, Barth E, Halpern H, Rosen GM, and Yuan CS (2002). Baicalein attenuates oxidant stress in cardiomyocytes. Am J Physiol Heart Circ Physiol 282:H999-H1006.

Shono $\mathrm{T}$, Ono M, Izumi H, Jimi SI, Matsushima K, Okamoto T, Kohno K, and Kuwano M (1996). Involvement of the transcription factor NF-kappaB in tubular morphogenesis of human microvascular endothelial cells by oxidative stress. Mol Cell Biol 16:4231-4239.

Singh AK, Seth $\mathrm{P}$, Anthony $\mathrm{P}$, Husain MM, Madhavan S, Mukhtar H, and Maheshwari RK (2002). Green tea constituent epigallocatechin-3-gallate inhibits angiogenic differentiation of human endothelial cells. Arch Biochem Biophys 401:2937.

Szatrowski TP and Nathan CF (1991). Production of large amounts of hydrogen peroxide by human tumor cells. Cancer Res 51:794-798.

Terao J (1999). Dietary flavonoids as antioxidants in vivo: Conjugated metabolites of (- )-epicatechin and quercetin participate in antioxidative defense in blood plasma. $\mathrm{J}$ Med Invest 46:159-168.

Tosetti F, Ferrari N, De Flora S, and Albini A (2002). Angioprevention: Angiogenesis is a common and key target for cancer chemopreventive agents. FASEB J 16:2-14.

Van Wetering S, van Buul JD, Quik S, Mul FP, Anthony EC, ten Klooster JP, Collard JG, and Hordijk PL (2002). Reactive oxygen species mediate Rac-induced loss of cell-cell adhesion in primary human endothelial cells. J Cell Sci 115:18371846.

Vittet D, Prandini MH, Berthier R, Schweitzer A, MartinSisteron H, Uzan G, and Dejana E (1996). Embryonic stem cells differentiate in vitro to endothelial cells through successive maturation steps. Blood 88:3424-3431.
Wang R, Clark R, and Bautch VL (1992). Embryonic stem cell-derived cystic embryoid bodies form vascular channels: An in vitro model of blood vessel development. Development 114:303-316.

Wartenberg M and Acker $H$ (1995). Quantitative recording of vitality patterns in living multicellular spheroids by confocal microscopy. Micron 26:395-404.

Wartenberg M, Donmez F, Ling FC, Acker H, Hescheler J, and Sauer $\mathrm{H}$ (2001a). Tumor-induced angiogenesis studied in confrontation cultures of multicellular tumor spheroids and embryoid bodies grown from pluripotent embryonic stem cells. FASEB J 15:995-1005.

Wartenberg M, Fischer K, Hescheler J, and Sauer H (2000). Redox regulation of $\mathrm{P}$-glycoprotein-mediated multidrug resistance in multicellular prostate tumor spheroids. Int $J$ Cancer 85:267-274.

Wartenberg M, Gunther J, Hescheler J, and Sauer H (1998). The embryoid body as a novel in vitro assay system for antiangiogenic agents. Lab Invest 78:1301-1314.

Wartenberg M, Ling FC, Schallenberg M, Baumer AT, Petrat K, Hescheler J, and Sauer H (2001b). Down-regulation of intrinsic P-glycoprotein expression in multicellular prostate tumor spheroids by reactive oxygen species. J Biol Chem 276:17420-17428.

Wong IY, He ZD, Huang Y, and Chen ZY (2001a). Antioxidative activities of phenylethanoid glycosides from Ligustrum purpurascens. J Agric Food Chem 49:3113-3119.

Wong IY, Huang Y, He ZD, Lau CW, and Chen ZY (2001b). Relaxing effects of Ligustrum purpurascens extract and purified acteoside in rat aortic rings. Planta Med 67:317-321.

Yoon SO, Park SJ, Yoon SY, Yun CH, and Chung AS (2002). Sustained production of $\mathrm{H} 2 \mathrm{O} 2$ activates pro-matrix metalloproteinase-2 through receptor tyrosine kinases/ phosphatidylinositol 3-kinase/NF-kappa B pathway. J Biol Chem 277:30271-30282.

Zhang AQ, Chan PT, Luk YS, Ho WKK, and Chen ZY (1997). Inhibitory effect of purified green tea epicatechin isomers on LDL-oxidation. J Nutri Biochem 8:334-340.

Zhang G, Miura Y, and Yagasaki K (2000). Suppression of adhesion and invasion of hepatoma cells in culture by tea compounds through antioxidative activity. Cancer Lett 159: 169-173. 